\title{
Autotrasplante renal laparoscópico experimental como modelo de aprendizaje de técnica laparoscópica
}

\author{
Aguilera Bazán A*, Betancourt F*, Murillo $\mathrm{S}^{* *}$, Benito de la Víbora J**, Cisneros Ledo J*, \\ de la Peña Barthel J*. \\ *Servicio de Urología. Hospital Universitario La Paz. Madrid. \\ **Facultad de Veterinaria. Universidad Complutense. Madrid.
}

Actas Urol Esp. 2008;32(1):160-165

\section{RESUMEN}

\section{AUTOTRASPLANTE RENAL LAPAROSCÓPICO EXPERIMENTAL COMO MODELO DE APRENDIZAJE DE TÉCNICA LAPAROSCÓPICA}

Introducción: La cirugía laparoscópica requiere un largo proceso de aprendizaje en el que se va aumentando progresivamente el grado de complejidad.

Objetivo: La técnica aquí presentada se ha realizado buscando un modelo experimental que nos permita realizar sutura vascular laparoscópica, de manera que el objetivo inmediato no es conseguir un autotrasplante funcionante, sino realizar con éxito anastomosis vasculares. Presentamos nuestra experiencia en los dos primeros casos realizados.

Material y método: Se realizó el autotrasplante renal laparoscópico en hembras de cerdo de entre 15 y 20 kg. El Servicio de Cirugía Experimental del Hospital Universitario La Paz revisó y aprobó los protocolos del experimento, tal como exigen los Dictámenes Europeos para la protección de los animales utilizados con fines científicos y experimentales (86/609/EEC).

Resultados: Al sacrificar a los animales, sólo observamos un caso con trombosis vascular arterial. E1 resto de animales presentaban buen flujo arterial y venoso.

Conclusiones: El autotrasplante renal laparoscópico experimental proporciona un buen modelo para aprendizaje de cirugía laparoscópica. Creemos que aporta recursos laparoscópicos adecuados para cirugía retroperitoneal y pélvica. No proponemos, en el momento actual, su aplicación a la cirugía laparoscópica en humanos.

Palabras clave: Autotrasplante renal. Entrenamiento laparoscópico. Laparoscopia vascular. Irrigación.

\section{ABSTRACT}

EXPERIMENTAL LAPAROSCOPIC RENAL AUTOGRAFT AS LEARNING LAPAROSCOPIC MODEL

Introduction: Laparoscopic surgery requires a long training period of time in which the complexity of the training is increased.

Goals:The technique presented in this paper has been developed in order to find an experimental model that allows us to improve the learning of the vascular suture. Our main goal was to evaluate this technique as an experimental model for the vacular anastomosis, not to obtain a functional autotransplant. In this regard, here we summarize our experience during the first two cases performed.

Material and methods: Laparoscopic renal autotransplant was perfomed in female lab pigs weighing 1520 kg. International Experimental Animal Care rules were accomplished.

Results: After sacrifizing the animals, only one case of vascular thrombosis was observed. The other cases showed normal arterial and venous flow.

Conclusions: Experimental Renal Laparoscopic autotransplant constitutes a good surgical model. We are trying to implement the technique in the clinics in the next future.

Keywords: Renal autograft. Training laparoscopy. Vascular laparoscopy. Irrigation. 
$\mathrm{E}^{\mathrm{n}}$ n los últimos años el panorama de la técnica quirúrgica abierta en Urología ha cambiado drásticamente con el desarrollo e implantación de la cirugía laparoscópica. El hecho de que la prostatectomía radical laparoscópica se haya convertido en una técnica reproducible aunque exigente, con unos resultados más que aceptables en cuanto a tiempo quirúrgico, estancia hospitalaria, complicaciones postoperatorias y control oncológico ha hecho que los urólogos empecemos a mirar a la laparoscopia no como una técnica limitada a la patología renal sino como una alternativa a la prostatectomía radical abierta, con todo lo que esto supone en el volumen anual de pacientes quirúrgicos de un servicio de Urología. Como muestra de este enorme interés, basta con observar la gran cantidad de cursos prácticos que han proliferado en nuestro país a propósito de dicha técnica.

Las principales dificultades que el cirujano se encuentra al iniciarse en laparoscopia son varias: pérdida de la visión tridimensional, pérdida del tacto (la laparoscopia asistida por la mano suple esta dificultad), dificultosa coordinación entre el ojo y la mano (vamos a operar sin ver nuestras manos), limitaciones tecnológicas del instrumental desarrollado que todavía está en plena evolución y además habría que añadir que supone no poder realizar muchas de las maniobras aprendidas en la cirugía convencional, lo que mermará nuestra capacidad $^{1,2}$. Todo esto hace que el aprendizaje sea laborioso $\mathrm{y}$, si bien no hay una pauta única a seguir, es cierto que el sentido común marca lo que debe ser este proceso.

Creemos que el desarrollo de esta capacidad quirúrgica debe pasar por un entrenamiento previo y exhaustivo en los sistemas de simulación quirúrgica (endotrainer) que permitirán adquirir familiaridad con el instrumental y con la diferente visión espacial que requiere esta técnica ${ }^{3}$. El siguiente paso debe ser el trabajo en modelos animales vivos en el que pondremos en práctica la técnica de generación del neumoperitoneo, colocación de trócares, disección de tejidos y desarrollo de la técnica quirúrgica propiamente dicha. Pensamos que previo a este paso con animales de experimentación se debe dominar la sutura laparoscópica en el endotrainer, de tal manera que el gasto económico que realizamos con el animal lo podamos aprovechar al máximo realizando todo tipo de ejercicios de disección y sutura.
En este sentido, el modelo de experimentación que presentamos en este artículo conjuga la disección delicada de tejidos con la sutura vascular. Se trata de un ejercicio quirúrgico muy exigente que requiere haber dominado la fase previa con endotrainer y la adecuada coordinación con el ayudante. El objetivo final no es la consecución de un autotrasplante funcionante, sino la realización de una sutura vascular exitosa como sistema de aprendizaje de técnica laparoscópica.

Este modelo se llevó a cabo hace aproximadamente 3 años en el Quirófano Experimental del Hospital La Paz con la colaboración de los veterinarios de dicho centro. En el momento actual el autor del artículo ha llevado a cabo aproximadamente 200 cirugías laparoscópicas en humano (retroperitoneales y prostáticas) de manera que lo que se describe es una visión retrospectiva de la técnica de aprendizaje seguida.

\section{MATERIAL Y MÉTODOS}

Se realizó el autotrasplante renal laparoscópico en hembras de cerdo de entre 15 y $20 \mathrm{~kg}$. El Servicio de Cirugía Experimental del Hospital Universitario La Paz revisó y aprobó los protocolos del experimento, tal como exigen los Dictámenes Europeos para la protección de los animales utilizados con fines científicos y experimentales $(86 / 609 / \mathrm{EEC})$.

\section{Técnica quirúrgica}

Con el animal colocado en decúbito lateral izquierdo (inicialmente elegimos el riñón derecho ya que el izquierdo tiene más probabilidad de tener más de una vena, lo que dificultaría enormemente la anastomosis venosa) realizamos el neumoperitoneo con aguja de Veress pinchando a nivel umbilical. Se trabajó con presiones intraabdominales de 12-13 mmHg. Procedemos a la colocación de los trócares como lo haríamos en el humano, buscando la triangulación entre ellos de manera que la cámara quede localizada entre las manos del cirujano. Comenzamos la disección abriendo el peritoneo por el polo inferior renal hasta identificar el uréter que nos llevará al hilio renal, si bien esta maniobra no es necesaria en estos animales dada la escasa presencia de grasa. Es importante no liberar la cara posterior renal, ya que de esta manera el riñón se encon- 
trará más fijo y esto nos facilitará la posterior canulación de la arteria renal. Una vez disecado el hilio renal con la máxima longitud de arteria y vena renal, se procede al clampaje de los mismos con clips laparoscópicos (animales de este tamaño no requieren más de un clip y la vena generalmente no requiere el uso de Endo GIA) A continuación, con tijera laparoscópica recta se procede a hacer una pequeña apertura en la cara anterior de la arteria renal, sin llegar a seccionarla del todo, que nos permitirá canularla para realizar la irrigación del riñón. Esta irrigación la realizaremos con un Abbocath del 24 al que le conectaremos el sistema de irrigación y procederemos a la instilación de Eurocollins previamente enfriado en nevera. El Abbocath con la conexión de irrigación lo introduciremos a través de un cuarto puerto de $10 \mathrm{~mm}$ colocado entre los puertos de nuestras manos. Se canula la arteria renal y se comienza a irrigar el riñón, mientras realizamos la apertura de la vena renal previamente clipada y referenciamos su luz con un Prolene de 7/0. La irrigación durará aproximadamente 15-20 minutos, hasta que se obtenga por la vena liquido de irrigación. Seccionamos la arteria y la espatulamos.

En la siguiente fase se realiza la disección de vasos iliacos (común y externa) como preparación para el clampaje previo a la anastomosis procediéndose entonces a completar la disección del riñón y el uréter que permitirá su descenso y rotación para afrontar el hilio renal a los vasos iliacos. Para el clampaje vascular se pueden emplear pinzas vasculares laparoscópicas (Bulldogs) o, en su defecto utilizaremos vessel-loop con un clip laparoscópico que haga la función de fruncir el vesselloop al vaso.

La fase de la anastomosis vascular es probablemente la más compleja y la que requiere mayores recursos. Colocamos un nuevo trócar de $5 \mathrm{~mm}$ en la zona inguinal del animal, justo delante de la pata trasera, para la mano izquierda. Comenzamos clampando únicamente la vena iliaca externa; es el momento de administrar intravenosamente heparina a dosis de $1 \mathrm{ml} / \mathrm{kg}$. Incidimos la vena con un corte transversal realizado con pinzas rectas, teniendo en cuenta que se trata de vasos de aproximadamente 3-4 mm de diámetro, lo que dificulta enormemente cualquier maniobra (Figs. 1 y 2). Identificamos la vena renal, que se suele retraer, gracias al punto de referen-

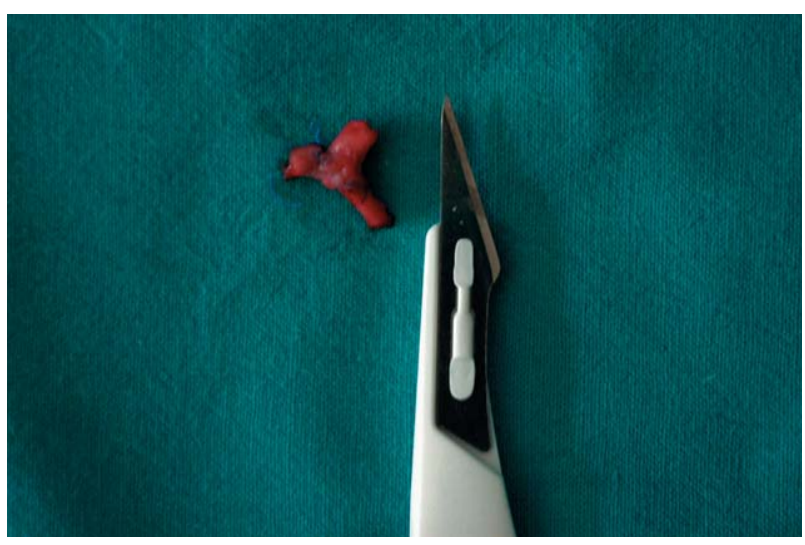

FIGURA 1. Anastomosis iliaca-arteria renal.

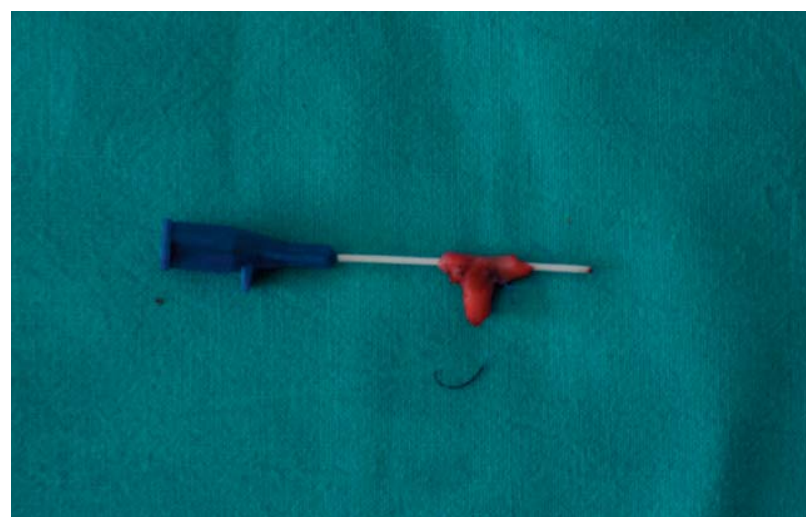

FIGURA 2. Calibre de anastomosis iliaca-arteria renal.

cia dado previamente. La anastomosis vascular se realizará con dos suturas continuas de Prolene 7/0 (es conveniente evitar la aguja Visi Black ya que es más frágil y no es infrecuente que se rompa). Iniciaremos la sutura en la cara posterior de la vena, pasando la sutura por la cara interna, finalizando con la sutura continua de la cara anterior. Los primeros puntos son los más complejos ya que la vena renal se encuentra completamente retraída. La labor del ayudante será la de mantener con una pinza de agarre la vena renal próxima a la iliaca. Una vez terminada la sutura, se desclampa la vena iliaca. Seguidamente debe clamparse la arteria iliaca externa de la misma manera que la vena. La apertura se realizará de forma similar, dando un corte con tijera recta en la cara anterior. Se procede a la anastomosis arterial con dos suturas continuas de Prolene 7 / 0 empezando por la cara posterior y pasando la sutura por dentro. Finalmente se desclampa la arteria iliaca. No debe observarse fuga tras el desclampaje en la vena ni en la arteria. 
Una vez cerrados los trayectos de los trócares se despierta al animal y se le mantiene vivo para proceder a su sacrificio a los 5 días, analizándose entonces en Anatomía Patológica los riñones trasplantados.

\section{RESULTADOS}

Esta cirugía ha sido realizada por completo en dos animales, con un tiempo quirúrgico total de 8 horas cada una. El tiempo de isquemia caliente (desde el clampaje hiliar hasta la canulación de la arteria) fue de 5 minutos en el primer caso y 5,30 minutos en el segundo caso.

La vena iliaca externa estuvo clampada 3 horas en ambos casos. La diferencia estuvo en el clampaje de la arteria. En el primer caso se clampó al mismo tiempo que la vena y el clampaje total de la arteria fue de 4 horas (la sutura se realizó en aproximadamente 1 hora), produciéndose una parálisis de la extremidad inferior cuando se despertó al animal, que prácticamente no llegó a recuperar en el postoperatorio. En el segundo caso se varió la técnica y se realizó primero el clampaje y la anastomosis venosa, que es la más laboriosa (3horas), sin clampar la arteria iliaca. Posteriormente se realizó la anastomosis arterial en 1hora y 15 minutos. En el postoperatorio el animal movía con normalidad las extremidades posteriores.

Al sacrificar a los animales a los 5 días, el que tenía dificultad para movilizar la extremidad trasera derecha presentaba una trombosis de la arteria iliaca externa y de la arteria renal, habiéndose perdido el implante. En el segundo caso la arteria iliaca y renal presentaban latido y flujo.

\section{DISCUSIÓN}

La laparoscopia urológica se encuentra en pleno apogeo tanto en nuestro país como fuera de él. Muestra de ello es la gran acogida que tienen los cursos con cirugía en directo realizada por expertos de talla internacional así como los innumerables cursos experimentales que han proliferado en nuestra geografia. El auge de esta técnica quirúrgica se ha desarrollado de manera paralela al de otras especialidades como la cirugía y la ginecología. Sin embargo, la laparoscopia urológica no se asemeja a éstas en el hecho de que no existen en nuestra especialidad procedimientos relativamente sencillos y de realización frecuente, tales como la ligadura de trompas o la colecistectomía, que permiten un progresivo aprendizaje por parte del cirujano, antes de acometer procedimientos más complejos ${ }^{4,5}$. Además se ha postulado que el aprendizaje de las técnicas minimamente invasivas es más largo que el correspondiente a la cirugía abierta. En ello influiría el aprendizaje que se efectúa durante la ayudantía quirúrgica en cirugía abierta, que permite en un primer momento un mejor contacto con el campo quirúrgico, así como una mejor visión tridimensional de éste ${ }^{6}$.

Cuando analizamos el grado de dificultad de los distintos procedimientos susceptibles de realización mediante cirugía laparoscópica en urología, encontramos algunos de menor grado de dificultad (varicocelectomía, criptorquidia, ablación de quiste renal) un segundo nivel de grado medio (ureterolitotomía, pielolitotomía, pieloplastia), un nivel de alta dificultad (adrenalectomía, nefrectomía radical, nefroureterectomía y nefrectomía parcial). En el escalón superior de dificultad encontraremos la linfadenectomía retroperitoneal, la cistectomía radical y la prostatectomía radical $^{7}$. Esta última es, dentro de nuestra especialidad, uno de los procedimientos más frecuentes. La dificultad técnica para su realización mediante técnicas laparoscópicas radica principalmente en la disección dificil, (preservación del cuello vesical y nervios erectores) y la realización de sutura intracorpórea para la anastomosis vesicouretral. Otros procedimientos de dificultad media o alta, como la cirugía renal, además de una menor frecuencia conllevan un riesgo de lesión vascular con una evidente morbimortalidad asociada. Consecuencia de ello es el retraso comparativo sufrido por la laparoscopia en urología.

La curva de aprendizaje para la cirugía laparoscópica es un concepto insuficientemente definido. Podría considerarse la curva de aprendizaje como "el número de procedimientos que necesita realizar un cirujano para realizar la técnica con seguridad, de forma independiente y con resultados razonables" ${ }^{6}$. Se ha postulado que la curva de aprendizaje para la cirugía laparoscópica resulta mayor que para la cirugía convencional. En este sentido se han desarrollado diversos modelos de aprendizaje, de dificultad creciente, comenzando la instrucción en simuladores (endotrainer) y progresando en la enseñanza mediante cirugía en modelos animales o en cadáveres. Finalmente debe iniciarse, tras un periodo de ayudantía, con la cirugía en humanos, comenzando siempre por procedimientos de bajo grado de difi- 
cultad. El modelo que realizamos hace ya tres años con el autotrasplante renal trataba de conjugar la disección fina de tejidos (disección de hilio renal y de iliacas) con la sutura intracorpórea y debe considerarse como técnica de alta complejidad. Este artículo, tres años después y con una experiencia de aproximadamente 200 cirugias laparoscópicas en humano trata de analizar la viabilidad del modelo de aprendizaje y su potencial aplicación en las distintas fases del aprendizaje de la laparoscopia.

El tamaño del animal empleado en nuestro trabajo, hembras de entre 15 y $20 \mathrm{Kg}$, resulta pequeño, lo que se traduce en la presencia de vasos renales e iliacos de reducido diámetro que dificultan en gran medida la sutura vascular. Animales más grandes podrían facilitar la técnica sobre todo al comienzo del aprendizaje aunque es cierto que son más caros y plantean más problemas de manejo en quirófanos experimentales pequeños (jaulas mayores, mesas de quirófano, etc...). El hecho de elegir hembras como modelo experimental nos permite el entrenamiento en otras cirugías más sencillas que pueden ser realizadas posteriormente en el animal o en su cadáver y que requieren la presencia de útero, como la sutura vésico-ureteral en el que empleamos el útero como modelo de uréter. Asímismo, el instrumental empleado debe ajustarse al tamaño de la sutura empleada ya que en caso contrario, podemos ocasionar deformaciones en la aguja. Aún así, nosotros hemos sido capaces de realizar la técnica con porta-agujas de $5 \mathrm{~mm}$ en lugar de los de $3 \mathrm{~mm}$ que deberían haber sido empleados en animales de ese peso.

Otro aspecto a tener en cuenta es el sistema de enfriamiento para disminuir el tiempo de isquemia caliente. En nuestro caso hemos empleado la canulación de la arteria renal y su posterior irrigación con Eurocollins previamente enfriado a $4^{\circ} \mathrm{C}$. Los resultados obtenidos con esta técnica cumplen bien los objetivos en cuanto a la realización de las anastomosis vasculares, puesto que no se registran sangrados después del desclampaje y se observa un latido adecuado. Sin embargo, los resultados en cuanto a función renal resultan pobres, seguramente como consecuencia del sistema de enfriamiento usado. Para mantener la funcionalidad del riñón a lo largo del trasplante debería añadirse al sistema aquí empleado una fuente de enfriamiento externo consistente en una bolsa de extracción de pieza de laparoscopia con hielo finamente picado que se pudiera introducir a través del trócar o con la instilación de suero a $4^{\circ}$ en la que se introduciría el riñón. Al no ser el objetivo del trabajo la consecución de un autotrasplante funcionante, se optó por el otro sistema que disminuyera el tiempo quirúrgico. Sin embargo, éste es un aspecto fundamental al tratarse de un modelo de experimentación y no un modelo aplicable, según este diseño, a la cirugía en humanos.

Indudablemente, esta cirugía no es la técnica más adecuada para iniciarse en laparoscopia. Ha sido realizada en nuestro servicio tras un largo proceso de aprendizaje que comenzó con técnicas básicas (manejo de endotrainer, ejercicios de sutura) que van ganando en complejidad de forma escalonada (nefrectomía, sutura vesical, disección vascular, nefrectomia parcial, anastomosis vesicoureteral, colgajo vesical, anastomosis vesicouretral, neovejiga intestinal), pero ha resultado ser de gran ayuda a la hora de adquirir habilidad en el manejo del instrumental y de la visión espacial, así como en el ejercicio de disección vascular.

Como se ha indicado previamente, han transcurrido tres años desde la realización de este ejercicio práctico durante los cuales el autor ha llevado a cabo aproximadamente 200 intervenciones laparoscópicas, con especial dedicación a la cirugía laparoscópica del retroperitoneo. La valoración retrospectiva de este modelo de aprendizaje resulta, según nuestro criterio, muy positiva por varios motivos que expondremos a continuación.

El ejercicio propiamente dicho consta de una primera fase de disección de tejidos; preparamos el hilio renal con la disección y canulación de los vasos y disecamos los vasos iliacos para proceder a su clampaje y preparación. Aunque la disección de tejidos no será el objetivo fundamental del modelo, puesto que ya habrá sido suficientemente realizada en el modelo de nefrectomía en animales, habrá de realizarse de manera minuciosa, puesto que de ello dependerá la adecuada preservación de las estructuras vasculares sobre las que habremos de actuar seguidamente. La disección del pedículo vascular resultará también más compleja que en el modelo de nefrectomía y redundará en una mejor preparación para los procedimientos retroperitoneales (nefrectomía, cirugía suprarrenal), donde necesitamos un buen control vascular, especialmente en la más dificultosa disección de venas. 
La siguiente fase, la de la anastomosis vascular, consta de dos suturas continuas de Prolene de 6 - 7/0. Indudablemente se trata de una fase para afianzar la técnica de sutura intracorpórea, no para iniciarse en ella. Requiere de una gran coordinación con el ayudante, de tanta importancia en laparoscopia. Este momento de la cirugía se debe abordar con gran paciencia ya que el manejo de una aguja y sutura tan pequeña es realmente complejo. Aunque el aprendizaje de la sutura intracorpórea puede realizarse suficientemente mediante la utilización de modelos básicos (tipo endotrainer $^{8}$, el entrenamiento necesario para la realización de técnicas complejas se verá potenciado mediante la realización de técnicas de alta exigencia, como la que realizamos en el actual estudio experimental. En el diseño de un programa de cirugía renal laparoscópica, el adecuado manejo de la sutura vascular redundará en unos mejores resultados, acortamiento de tiempos quirúrgicos y reducción en el número de reconversiones a cirugía abierta, en aquellos casos en que se produzca una lesión vascular inadvertida.

Analizando a posteriori todo el modelo nos parece que nos ha aportado un manejo más preciso en la colocación de la aguja de sutura, un mejor "tacto" en la disección vascular, nos ha permitido ser más conscientes de la importancia del ayudante en esta técnica y algo realmente dificil de adquirir como es la paciencia que requiere este tipo de cirugía, tanto enfocado a la cirugía del retroperitoneo como de la prostatectomía radical laparoscópica. No olvidemos que la prostatectomía radical laparoscópica en los comienzos de un programa es una técnica larga y que al final de la misma precisa de una anastomosis intracorpórea cuando habitualmente el equipo quirúrgico se encuentra más cansado. Haber entrenado esta situación resulta indudablemente beneficioso.

Las indicaciones para un autotrasplante renal son infrecuentes e incluyen diversas patologias tales como la patología vascular aorto-renal, lesiones ureterales extensas (tumores, traumatismos estenosis) y la fibrosis retroperitoneal ${ }^{9}$. Constituye una intervención quirúrgica compleja, con dos partes bien definidas: la nefrectomía "exvivo" y el autotrasplante, que precisan dos amplias incisiones. Su realización debe quedar reservada a urólogos con amplia experiencia, tanto en cirugía urológica como en suturas vasculares. El diseño del presente modelo laparoscópico de experimentación animal se realizó fundamentalmente con fines de aprendizaje y perfeccionamiento en sutura vascular y es en ese aspecto concreto en el que creemos que puede resultar de utilidad cuando lo revisamos con la perspectiva del tiempo. Para su aplicación como modelo de autotrasplante en humanos sería preciso desarrollar un modelo fiable de perfusión y conservación renal intracorpórea, que nos asegurara una adecuada preservación de la unidad renal, especialmente teniendo en cuenta la larga duración del procedimiento. Deberemos tener en cuenta además que las técnicas de experimentación animal, no siempre tienen aplicabilidad al humano; en ello influyen factores diversos como el diseño del modelo de experimentación o las diferencias anatómicas que han de ser siempre tenidas en cuenta.

Por lo tanto, pensamos que se trata de un buen modelo para aprendizaje de cirugía laparoscópica. Creemos que aporta recursos laparoscópicos adecuados para cirugía retroperitoneal y pélvica. No proponemos, en el momento actual, su aplicación a la cirugía laparoscópica en humanos.

\section{REFERENCIAS}

1. Sakti Das. Laparoscopia urológica avanzada. Clinicas Urológicas de Norteamérica. 2001;1(1-5).

2. Günter Janetschek, Jens Rassweiler. Cirugía laparoscópica en urología. Capítulo 6. Pag. 55-64. Ed.MASSON 1998.

3. Topel H.C. Endoscopio suturing and knot tying manual. Ethicon, Hamburg 1991.

4. Hunter, JG, Sackier, JM. Training for minimally invasive surgery. Mc Graw-Hill. New York. 1993.

5. John A.Libertino. Campbell’s Urology $7^{\text {a Ed }}$. Capítulo 12. Pag.460-489. Walsh, Retik, Vaughan, Wein. Ed.Saunders.

6. Subramonian K, DeSylva S, Bishai P, Thompson P, Muir G. Acquiring surgical skills: a comparative study of open versus laparoscopic surgery. Eur Urol. 2004;45(3):346-351.

7. Piechaud PT, Pansadoro A. Transfer of skills from the experimental model to the patients. Curr Urol Rep. 2006; $7(2): 96-99$.

8. Griffin S, Kumar A, Burgess N, Donaldson P. Development of laparoscopic suturing skills: a prospective trial. J Endourol. 2006;20(2):144-148.

9. Webster J, Lemoine J, Seigne J, Lockhart J Bowers V. Renal autotransplantation formanaging a short upper ureter or after ex vivo complex renovascular reconstruction. BJU Int. 2005;96(6):871-874.

10. Ames CD, Vanlangendonck R, Morrissey K, Venkatesh R, Landman J. Evaluation of surgical models for renal collecting system closure during laparoscopic partial nephrectomy. Urology. 2005;66(2):451-454.

Correspondencia autor: Dr. A. Aguilera Bazán

Servicio de Urología. Hospital La Paz

$\mathrm{P}^{\circ}$ de la Castellana 261 - 28046 Madrid

Tel.: 917277000

E-mail autor: aaguilera11@yahoo.es

Información artículo: Original 